\title{
Preliminary Evidence from a Cross-sectional Study on Epidemiology and Early Transmission Dynamics of COVID-19 in Karnataka State of India
}

\author{
Bakilapadavu Venkatraja ${ }^{1}$ \\ Madhura Nagesh Hegde ${ }^{4}$ \\ ${ }^{1}$ Department of Economics, Shri Dharmasthala Manjunatheshwara \\ Institute for Management Development, Mysuru, Karnataka, India \\ 2Department of Quantitative Methods, Shri Dharmasthala \\ Manjunatheshwara Institute for Management Development, \\ Mysuru, Karnataka, India \\ ${ }^{3}$ Division of Infectious Diseases, Nitte University Centre for \\ Science Education and Research, Nitte (deemed to be) University, \\ Deralakatte, Mangaluru, Karnataka, India \\ ${ }^{4}$ Department of Information Science and Engineering, Sahyadri \\ College of Engineering and Management, Mangaluru, Karnataka, \\ India
}

J Health Allied Sci Nu 2021;11:178-187.

\author{
Ballamoole Krishna Kumar ${ }^{3}$ \\ Ganaraj Karuvaje ${ }^{4}$ Praveen Rai ${ }^{3}$
}

\begin{abstract}
Address for correspondence Bakilapadavu Venkatraja, PhD, Department of Economics, Shri Dharmasthala Manjunatheshwara Institute for Management Development (SDMIMD), Mysuru 570 011, Karnataka, India (e-mail: venkatraja@sdmimd.ac.in)
\end{abstract}

Ballamoole Krishna Kumar, Division of Infectious Diseases, Nitte University Centre for Science Education and Research, Nitte (deemed to be) University, Deralakatte, Mangaluru 575018, Karnataka, India (e-mail: krishnakumarb@nitte.edu.in).

\begin{abstract}
Keywords

- COVID-19

- health status

- fatality

- CFR

- demography

- epidemiology

- Karnataka

Introduction Coronavirus disease 2019 (COVID-19) is an emerging infection and quickly disseminated around the world. This article studies the epidemiology and early transmission dynamics of COVID-19 in Karnataka, which would be useful for effective epidemic management and policy formulation.

Materials and Methods All COVID-19 cases reported in the state of Karnataka, India, till June 12, 2020, are included in the study. The epidemiology and transmission dynamics of COVID-19 in Karnataka is studied through descriptive statistical analysis.

Results The findings illustrate a gender-, age-, and region-based disparity in the susceptibility and fatality. There appears to be a male preponderance in the susceptibility, but a female preponderance in fatality. It is also found that the adults are more susceptible to the infection, while the elderly have the risk of high fatality. Further, infected individuals in the region with urbanization have a higher risk of fatality than other regions. The study shows that the chances of recovery for females are lower than males, and further, the chances of recovery are positively related to the age of the infected person. The chances of recovery are higher if the infected individual is younger and they diminish if the individual is older. The study also explores that the chances of recovery are affected by the patient's geographical location. It is also noted that individuals who returned from foreign travel have better chances of recovery than the locally transmitted individuals.
\end{abstract}

published online May 11, 2021

\section{May 11,2021}

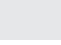

作


Conclusion Though the risk of susceptibility to COVID-19 infection is equal to all, the burden of getting infected and the burden of fatality is unequally distributed among different demographic categories. To manage the contagious spread of epidemic, to reduce fatality, and to increase the chances of recovery, targeted policy actions are suggested to benefit the vulnerable demographic categories.

\section{Introduction}

Coronavirus disease 2019 (COVID-19) is an emerging infectious disease caused by severe acute respiratory syndrome coronavirus 2 (SARS-CoV-2) that originated from the Chinese city of Wuhan in December 2019 and rapidly disseminated in most part of the world due to travel-associated activities. ${ }^{12}$ The disease spreads mostly through droplets released when an infected person coughs, speaks, or sneezes and the disease spreads rapidly among contacts. SARS-CoV-2 infection has a broad range of clinical manifestations varying from asymptomatic to symptomatic, including respiratory symptoms, fever, cough, dyspnea, and viral pneumonia. ${ }^{3-5}$ Based on the currently available pieces of evidence, it is noteworthy to mention that the severity of COVID-19 differs significantly within populations and geographical locations. ${ }^{5-7}$ As of now, there are no established vaccines or therapeutic options for COVID-19 and most infected patients have been treated with supportive care. ${ }^{7}$ In India, the first confirmed case of COVID-19 was reported in late January 2020 from an individual returned from Wuhan, and new cases of COVID-19 were subsequently reported in many Indian states. ${ }^{8}$ The interim analysis of the genomic data on Indian isolates of SARS-CoV-2 shows the introduction of this virus to India from multiple sources such as China, Europe, United States, Canada, and the Middle East. ${ }^{9-12}$ On March 8, 2020, more than a month after the first case was reported in India, Karnataka, a southern Indian state, reported its first COVID-19 case. Since then, the trajectory of COVID-19 cases in the state has grown abruptly despite the stringent interventions, including nationwide lockdowns to curb the COVID-19 outbreak. In the wake of the rising cases, the health department has implemented 5T strategy (trace, track, test, treat, and technology) under the Integrated Disease Surveillance Program (IDSP) to contain the spread of viral infection in the state. As of June 12,2020 , the state has reported 6,488 positive cases and final clinical outcomes were known for 3,788 cases, with 78 deaths and 3,710 recoveries. The disease outbreak did spread across 30 districts of the state, of which Kalburgi was the most affected district with $12.6 \%(n=818)$ cases. The mean age of infected patients was 31.2 years and the mean age of the deceased was 59.88 years. With this backdrop, this article studies the epidemiology and the early transmission dynamics of COVID-19 in Karnataka. The specific objectives are to study the pattern of fatality from COVID-19 across age, gender, and region and to identify the impact of demographic factors on the health status (HS) of the infected individuals.

\section{Materials and Methods}

\section{Study Area and Study Period}

The present study has been performed on the confirmed cases of COVID-19 in the state of Karnataka in India. The study covers all reported cases between March 9, 2020, and June 12, 2020.

\section{Data and Variables}

The study is based on publicly available data collected from https://www.covid19india.org/ and the COVID-19 information portal of the government of Karnataka (https:// covid19.karnataka.gov.in/english) on the confirmed cases of COVID-19 in Karnataka. The data, later, were curated, which includes information on daily and cumulative numbers of COVID-19 cases, recoveries, and deaths as well as the unit-level information on various cross-sections such as age, gender, travel history-based transmission type, and geographical location. The individual-level information was missing for $\sim 5 \%$ of the cases. For analytical purposes, as per the standard practice, it was assumed that missing information was similar to the information distribution of the rest of the $95 \%$ cases $^{13}$ and the missing information was distributed based on the mode value of the reported data.

\section{Sequence and Statistical Estimations}

The initial part of the article studies the epidemiology and transmission dynamics of COVID-19 in Karnataka through descriptive statistical analysis. The analysis is expected to bring forth age, gender, and travel-specific differentials in the burden of infection.

The study, in the later stage, estimates case fatality rate (CFR) across gender, age groups, and region for the entire data reported as of June 12, 2020, in Karnataka to visualize the dynamics of fatality occurring from COVID-19. The CFR denotes the risk of mortality from COVID-19 and estimated as the ratio of deaths $(\mathrm{Dt})$ to total infected cases $(\mathrm{Ct})$, as given in Equation 1.

$$
\mathrm{CFR}=\mathrm{Dt} / \mathrm{Ct}
$$

It is estimated for males and females at all age groups as well as for different regions and provides an analysis on the gender, age, and region-specific differentials in the burden of COVID-19 fatalities. However, the analysis based on CFR has a limitation. Since the epidemic is ongoing, the CFR may change as the probability of death among currently active cases is nonzero. ${ }^{13}$ Previous studies have shown that the preferential inclusion of the only cases reported to the health authorities, as well as the delay between the onset of the disease and the final outcome, can lead to biased CFR estimates, particularly during the 
exponential phase of the outbreak of the disease. ${ }^{14,15}$ Owing to this, this study, later, considers the likely mortality in the active cases as well, and estimates the adjusted CFR (CFR*) by following the method proposed by Joe and coworkers. ${ }^{13}$ Accordingly, the CFR* is measured by applying Equation 2 .

$$
\mathrm{CFR}^{*}=\emptyset(1+\alpha)
$$

In Equation 2, ø denotes CFR, that is estimated as a ratio of deaths to total cases ( $\mathrm{Dt} / \mathrm{Ct}$ ), and $\alpha$ refers to the ratio of active cases to total cases $(\mathrm{At} / \mathrm{Ct})$. The estimation assumes that the active cases will have same relative probabilities of death and recoveries. ${ }^{13} \mathrm{~A}$ divergence in CFR* curve from the CFR curve indicates that the epidemic is in the transmission phase. As we approach the end of the epidemic, the active cases of infection will constantly decline toward zero $(\alpha=0)$ and CFR* will be approaching toward CFR to converge. It is in this phase that the two curves would start moving closer and converge at one point in time, denoting the end of the epidemic.

The insights of CFR and CFR* is limited to visualization of rate of fatality across age, gender, and region, and it does not reflect the role of the demography of the individual on the chances of change in the health status. And hence, in the next stage of analysis, a cross-sectional disaggregated analysis has been deployed by estimating a logistic regression model to explore the differentials in the predicted chances of an infected individual surviving the COVID-19. It analyses the impact of different categories of various demographic factors such as age, gender, transmission type, and geography on the HS of the individual. The general form of the estimated model is presented in Equation 3.

Health status $=\mathrm{f}($ gender, age, transmissiontype, geography $)\{3\}$

The variables are classified into different categories according to their demographic nature. The HS of an individual is a binary variable that reflects the chances of the infected individual getting recovered or being deceased from COVID-19. Gender forms whether the infected individual is male or female. On the basis of the age, the infected individuals are grouped into categories such as children (between 0 and 14 years [age 1]), youth (between 15 and 44 years [age 2]), older adults (between 45 and 64 years [age 3]), and elders (65 years and older [age 4]). Though initially the COVID 19 was detected only among the individuals having foreign travel history, at the later stage, transmission among individuals locally also accelerated. So, the study considers both the types of COVID-19 transmissions. The state of Karnataka is characterized with geoenvironmental differences and accordingly for effective administrative purposes four administrative divisions are created by the government. They are geo 1-Belgaum division (covering districts such as Bagalkote, Belgaum, Vijayapura, Dharwad, Gadag, Haveri, and Uttara Kannada), geo 2-Gulbarga division (Ballari, Bidar, Gulbarga, Koppala, Raichuru, and Yadagiri), geo 3-Mysuru division (Chamarajanagara, Chikkamagaluru, Dakshina Kannada, Hassan, Kodagu, Mandya, Mysuru, and Udupi), and geo 4-Bengaluru division (Shimoga, Bengaluru Rural, Ramanagara, Chikkaballapura, Chitradurga, Davanagere, Kolar, Bengaluru Urban, and Tumakuru). Some of the travelers from other states are also found positive in Karnataka and this study develops a fifth geographical division (Geo 5) of "other states" to the analysis.

The model defines that the odds of survival or decease of an infected person is governed by whether they are male or female, the age group to which (s)he belongs, whether the transmission is local or imported, and to which geographical region (s)he belongs.

Since the factors are categorical, adoption of a logistic regression model with dummy variables appears to be the most appropriate statistical technique. It estimates whether the differences in the demographic profile of infected persons contribute to differential health status. The dummy variables that are considered in the study are defined as follows.

DGender $=1$ if the individuals gender is male, 0 if gender is female.

DAge $1=1$ if the individuals age is 0 to 14 years; = 0 otherwise.

DAge $2=1$ if the individuals age is 15 to 44 years; = 0 otherwise.

DAge $3=1$ if the individuals age is 45 to 64 years; = 0 otherwise.

DAge $4=1$ if the individuals age is 65 to 100 years; = 0 otherwise.

DTrans = 1 if the individuals transmission type is imported; 0 if the transmission type is local.

DGeo1 = 1 if the individual belongs to geography division 1 ; $=0$ otherwise.

DGeo2 = 1 if the individual is belongs to geography division 2; $=0$ otherwise.

DGeo3 = 1 if the individual is belongs to geography division $3 ;=0$ otherwise.

DGeo4 $=1$ if the individual is belongs to geography division $4 ;=0$ otherwise.

DGeo5 $=1$ if the individual is belongs to geography division $5 ;=0$ otherwise

The study considers female gender, age 4, local transmission, and geo 5 as the base category for further analysis. Given the base category as specified, the final model estimated in the present study is as given in Equation 4.

$$
\begin{aligned}
\log \left(\frac{P(\text { recovery })}{1-P(\text { recovery })}\right) & =\beta_{0}+\beta_{1} D \text { Gender } 1+\beta_{2} D \text { Age } 1+ \\
& \beta_{3} D \text { Age } 2+\beta_{4} D \text { Age } 3+\beta_{5} D \text { Trans } 1+ \\
& \beta_{6} D G e o 1+\beta_{7} D G e o 2+\beta_{8} D G e 03+ \\
& \beta_{9} D G e 04
\end{aligned}
$$

Where, $\log \left(\frac{P(\text { recovery })}{1-P(\text { recovery })}\right)$ is the response variable in

the model which measures the HS of the infected individual and it reflects the odds of him/her either getting recovered or deceased; $P$ (recovery) is the probability of the infected individual recovering; $1-P($ recovery $)=P$ (deceased $)$ denotes the probability of the mortality of the infected individual; $\beta_{0}$ is the intercept of the model which measures the HS of the individuals in the base category; and $\beta_{1}, \beta_{2}, \beta_{3}, \beta_{4}, \beta_{5}, \beta_{6}, \beta_{7}, \beta_{8}$, and $\beta_{9}$ are the coefficient values of their respective regressors. 


\section{Results}

The outcome of the curve-fitting exercise for the COVID-19 data in Karnataka has been depicted in - Fig. 1. It reveals the temporal trend in the incidence of COVID-19 cases from March 9, 2020, to June 12, 2020. From the trend analysis, it is clear that the number of COVID-19 cases in Karnataka was greatly suppressed during the lockdown period, but the state had seen an increase in the number of COVID-19 cases immediately after the relaxations in the lockdown norms. It is evident from the trends in cases reported daily.

It could be noted from $\boldsymbol{- F i g} \mathbf{1}$ that there was a higher incidence of infection in males, with a cumulative male to female ratio of 1:0.59. A gender-specific analysis (-Table $\mathbf{1}$ ) also brings forth that in each of the age categories, the burden of getting infected is more on males than females. If the gender difference is less among children and elderly, it is wide among middle-age groups (youths and older adults) and more favorable to females. Mann-Whitney U test (two-tailed) confirms that there is a difference between males and females in the susceptibility to COVID-19 infection, which is statistically significant ( $p$-value of $U$ critical $=0.012$ ).

In this analysis, age-varying susceptibility in COVID-19 was observed, and the youth age group (15-44 years) in both the genders has a combined burden of $~ 65 \%$ to the total number of COVID-19 cases (-Table 1). Children account for $15 \%$ of the total reported cases of COVID-19 in Karnataka, compared with $17 \%$ in the 45 to 64 years age groups of older adults.

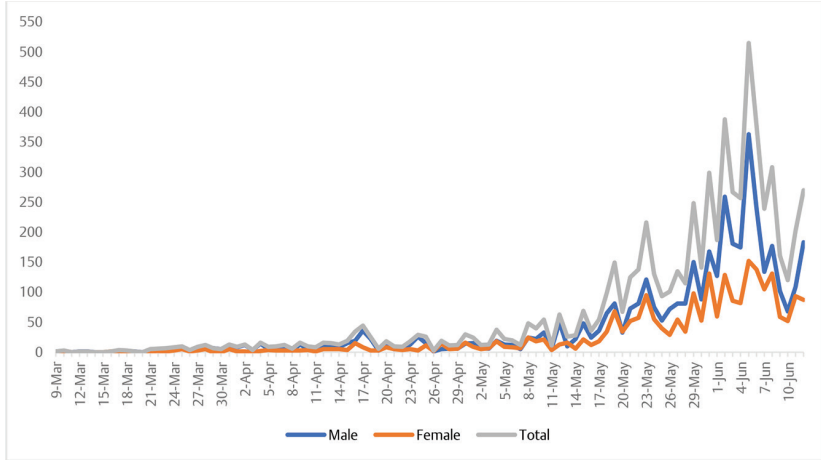

Fig. 1 Trends in transmission growth of coronavirus disease 2019 (COVID-19) (daily) in Karnataka State.
Meager proportions (3\%) of COVID-19 cases have been reported among elderly citizens in Karnataka during the study period compared with the incidence rate of over $14 \%$ in older persons as reported from various high-income countries. ${ }^{16-18}$ Further, the adult population aged between 15 and 64 years has a relatively very high burden of $\sim 82 \%$ in total reported positive cases ( - Fig. 2 ). Hence, there are differences in the susceptibility to the infection between age groups and such differences in the number of COVID-19-infected cases between the different age groups is statistically significant ( $p$-value $=0.0001)$.

People among the infected population are predominately without a history of foreign travel (-Table 2). Only $2.68 \%$ of the infected people have a record of international travel and more than $97 \%$ cases are acquired locally. In the cases of both local and imported transmissions, as found in the overall category, the age-wise and gender-wise infection pattern has a male and youth preponderance. It is found from the analysis that since mid-May 2020, with the beginning of international flights, the positive cases among the foreign-returned individuals started increasing ( - Figs. 3 and 4). The Mann-Whitney U test (two-tailed) confirms that there exists a statistically significant difference in the number of COVID-19-infected cases identified between people with foreign travel and domestic travel $(p=0.0001)$.

\section{Dynamics of Fatality}

- Table 1 presents the age-specific relative burden in the mortality from COVID-19 among males and females in

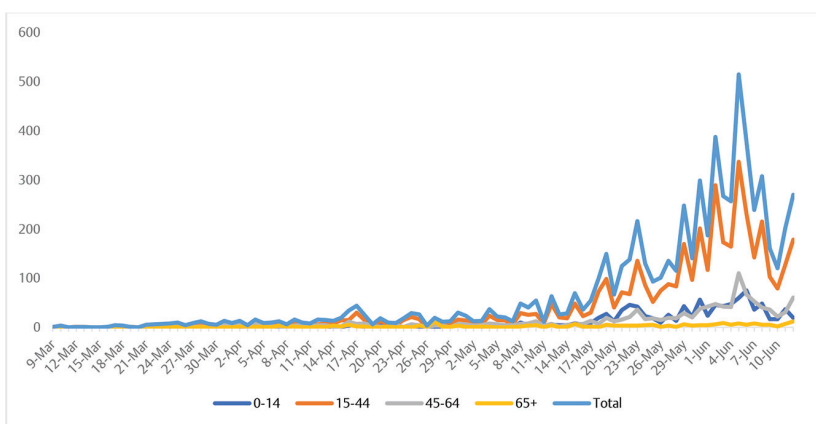

Fig. 2 Age-specific trends in coronavirus disease 2019 (COVID-19) cases (daily data in numbers) in Karnataka.

Table 1 Gender-specific coronavirus disease 2019 (COVID-19) cases and deaths by age groups in the Indian state of Karnataka

\begin{tabular}{|l|l|l|l|l|l|l|}
\hline \multirow{2}{*}{ Age group (y) } & \multicolumn{3}{|c|}{ Cases } & \multicolumn{3}{c|}{ Deaths } \\
\cline { 2 - 7 } & Male & Female & Total & Male & Female & Total \\
\hline $0-14$ & $540(13.24)$ & $440(18.27)$ & 980 & - & $1(3.03)$ & 1 \\
& {$[8.32]$} & {$[6.78]$} & {$[15.10]$} & & {$[1.28]$} & {$[1.28]$} \\
\hline $15-44$ & $2681(65.71)$ & $1507(62.58)$ & 4188 & $6(13.33)$ & $3(9.09)$ & 9 \\
& {$[41.32]$} & {$[23.23]$} & {$[64.55]$} & {$[7.69]$} & {$[3.84]$} & {$[11.53]$} \\
\hline $45-64$ & $749(18.36)$ & $360(14.95)$ & 1109 & $20(44.44)$ & $16(48.48)$ & 36 \\
& {$[11.54]$} & {$[5.55]$} & {$[17.09]$} & {$[25.64]$} & {$[20.51]$} & {$[46.15]$} \\
\hline+65 & $110(2.70)$ & $101(4.19)$ & 211 & $19(42.22)$ & $13(39.39)$ & 32 \\
& {$[1.7]$} & {$[1.56]$} & {$[3.25]$} & {$[24.36]$} & {$[16.66]$} & {$[41.02]$} \\
\hline All & $4080[62.9]$ & $2408(37.1]$ & 6488 & 45 & $33[42.30]$ & 78 \\
& & & {$[100)$} & {$[57.70]$} & & {$[100]$} \\
\hline
\end{tabular}

Note: Data in () is percentage of male or female; data in [ ] is percentage of total. 
Karnataka. The result manifests that the burden of risk of mortality among children is near zero $(0.10 \%)$ and it increases as we proceed to the higher age groups. As per the data available till June 12 , though children account for $15 \%$ to the total infection, the share in the mortality is only $1.28 \%$. It is the

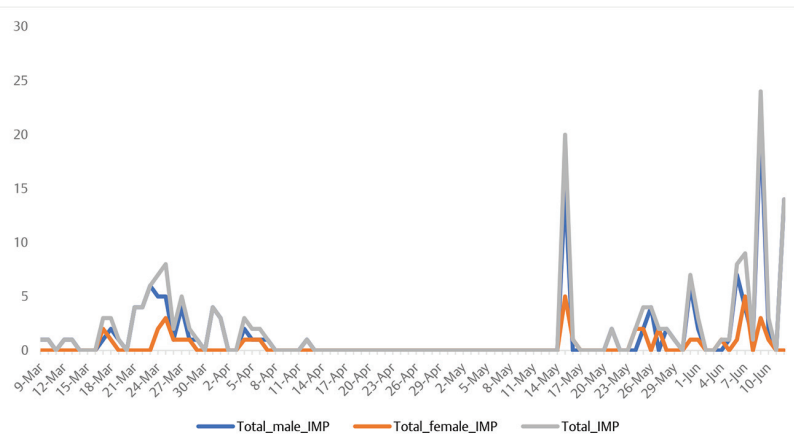

Fig. 3 Infection cases among people returned from abroad (gender-wise). (Note: IMP = imported cases).

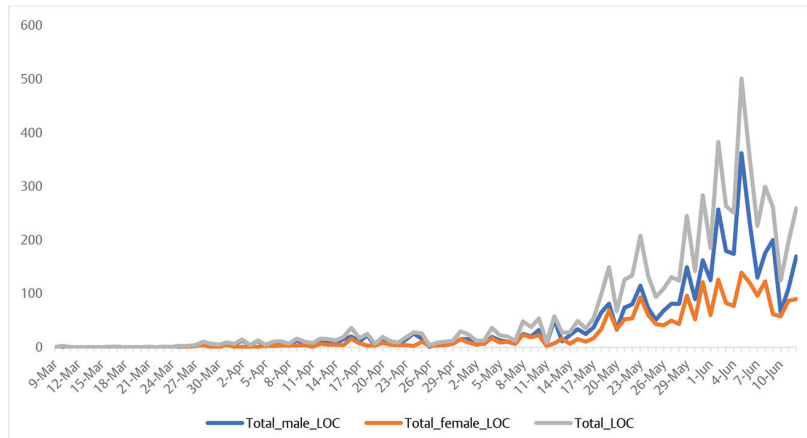

Fig. 4 Infection cases among domestic residents with no foreign travel history (gender-wise). (Note: LOC = locally acquired cases). indication that though children are susceptible to the infection, the severity of the infection appears to be mild among them in both the genders. The youth age group accounts for $11.5 \%$ of the total deaths due to COVID-19 in Karnataka as against $64 \%$ of the infected people who belonged to this age group. Though the share of the mortality in this age group is slightly higher than the children, it is much lesser in relation to its share in the burden of total infection. The burden of death is relatively very high among the infected people who are older than 45 years, accounting for $86.17 \%$ of the total COVID-19 deaths. However, the share of this group to the total infections is only $20.34 \%$. Thus, it is evident from the results that the youth age group has a higher burden of the infections, while the burden of death is relatively very high on the aged population. A gender-wise analysis also provides a similar pattern that males have more fatality than females across all age groups except children.

The CFR of Karnataka, as of June 12, 2020, is estimated to be $1.2 \%$ (-Table 3 ). This appears to be lower than the national CFR of India as well as other COVID-19-affected countries (China, United States, Italy, France, and Brazil among others). The CFR is very low $(0.21 \%)$ among children and youth $(0.10 \%$ and $0.21 \%$, respectively). Among the older adults, it is slightly higher (3.24\%) than the children and youth. The elderly population (older than 65 years) who are infected with the COVID-19 have a highly elevated risk of mortality (15.16\%). CFR of COVID-19 varies widely between different countries over a period of time. According to the study of Mi et al, ${ }^{19}$ the average CFR in China was $6.1 \pm 2.9 \%$ in 82,735 confirmed cases as on April 18, 2020. As on March 26, 2020, the overall CFR due to COVID-19 in Italy was $9.2 \%{ }^{19}$

The gender-wise estimation of CFR indicates that as of June 12, 2020, in Karnataka, females (1.37\%) have a relatively higher risk of mortality from COVID-19 than males (1.10\%).

Table 2 Age-wise and gender-wise distribution of the local and imported cases (percentage of total cases) in Karnataka

\begin{tabular}{|l|l|l|l|l|l|l|}
\hline \multirow{2}{*}{ Age group (y) } & \multicolumn{2}{|l|}{ Imported } & \multicolumn{2}{l|}{ Local } \\
\cline { 2 - 8 } & Male & Female & Total & Male & Female & Total \\
\hline $0-14$ & 0.030 & 0.030 & 0.06 & 8.18 & 6.19 & 14.36 \\
\hline $15-44$ & 1.56 & 0.29 & 1.85 & 40.93 & 22.80 & 63.74 \\
\hline $45-64$ & 0.30 & 0.20 & 0.51 & 11.33 & 5.44 & 16.77 \\
\hline $65+$ & 0.18 & 0.08 & 0.26 & 1.44 & 1.01 & 2.45 \\
\hline Total & 2.08 & 0.60 & 2.68 & 61.88 & 35.44 & 97.32 \\
\hline
\end{tabular}

Table 3 Age- and gender-specific coronavirus disease 2019 (COVID-19) case fatality rate (CFR) (in percentage) in Karnataka, up to June 12,2020

\begin{tabular}{|c|c|c|c|c|c|c|c|c|c|}
\hline $\begin{array}{l}\text { Age } \\
\text { group (y) }\end{array}$ & CFR-male & $\begin{array}{l}\text { Lower } \\
\text { limit }\end{array}$ & $\begin{array}{l}\text { Upper } \\
\text { limit }\end{array}$ & $\begin{array}{l}\text { CFR- } \\
\text { female }\end{array}$ & $\begin{array}{l}\text { Lower } \\
\text { limit }\end{array}$ & $\begin{array}{l}\text { Upper } \\
\text { limit }\end{array}$ & CFR-total & $\begin{array}{l}\text { Lower } \\
\text { limit }\end{array}$ & $\begin{array}{l}\text { Upper } \\
\text { limit }\end{array}$ \\
\hline $0-14$ & 0.00 & 0.00 & 0.00 & 0.22 & 0.01 & 1.25 & 0.10 & 0.00 & 0.56 \\
\hline $15-44$ & 0.22 & 0.08 & 0.48 & 0.19 & 0.04 & 0.58 & 0.21 & 0.09 & 0.40 \\
\hline $45-64$ & 2.67 & 1.6 & 4.1 & 4.44 & 2.56 & 7.11 & 3.24 & 2.28 & 4.46 \\
\hline $65+$ & 17.27 & 10.7 & 25.7 & 12.87 & 7.03 & 21.00 & 15.16 & 10.61 & 20.73 \\
\hline Total & 1.10 & 0.81 & 1.47 & 1.37 & 0.94 & 1.91 & 1.20 & 0.95 & 1.49 \\
\hline
\end{tabular}


The CFR is higher for males in all age groups except for children ( $0-14$ years) and older adults (45-64 years). It is in the older adult age group, females have a greater disadvantage of survival over males, and this probably contributes to a marginally higher overall risk of COVID-19-related mortality for females.

The temporal analysis of the CFR of Karnataka shows that with each month, the CFR is declining, which is particularly evident from the month of June, 2020. This trend could be attributed to a substantial rise in the infected cases and the HS of nearly one-third of the infected cases was "active" and were not categorized into either recovered or deceased as it happens only after few days. The epidemic is underway, and this would have caused a deflationary effect on the CFR.

When the epidemic is underway, the CFR* is a better tool of assessing the fatality as it considers the active cases, unlike CFR; hence, the CFR* is estimated for Karnataka in this study and the results are displayed in - Table 4 . The CFR* reflects a similar pattern in the distribution of the burden of mortality across age and gender as that of CFR. The CFR* for males has been estimated at $1.56 \%$ against $1.1 \%$ of CFR. Even among females, the CFR* increased to $1.93 \%$ from $1.37 \%$ of CFR. As of June 12,2020 , the CFR* for Karnataka is estimated at $1.7 \%$, as against $1.2 \%$ of CFR. Higher CFR* over CFR appears to be mainly accounted for sharp rise in CFR* of elderly people (older than 65 years) over their CFR. This is evident from the widened gap between CFR and adjusted CFR in the elderly age group. This denotes that even among the active cases, the predicted risk of mortality in elderly people is high.

The temporal analysis shows that the total CFR* is inclined to rise after May 2020 and with the time CFR* deviates widely from the CFR. The divergence between the CFR and CFR* curves ( - Fig. 5) is the indication that the COVID-19 in Karnataka is in the early transmission phase. Unless a drastic fluctuation in the infection cases or in the fatality numbers, the COVID-19 related mortality will be within the range of CFR and CFR*. As the epidemic transmission declines, the two curves would start moving closer, and converge at one point in time, indicating the end of the epidemic.

The study also unravels ( $\mathbf{- T a b l e ~} \mathbf{5}$ ) wide differences in the burden of fatality of COVID-19 between the geographical divisions of Karnataka. Though people travelled from other states to Karnataka have the highest fatality rate, the number of cases and the deceased is negligible in relation to the other four divisions. It is denoted that the Bengaluru division has a very high rate of fatality and the predicted risk of mortality (CFR ${ }^{*}$ ) in this division is also higher than in other divisions. The Mysuru division has the lowest CFR and CFR*. If the Gulbarga division has a satisfactory level of fatality, the predicted risk of mortality of COVID-19 infected individuals in Belgaum division is slightly higher. Thus, it is evident that there is a wide regional disparity in the risk of mortality from COVID-19.

\section{Demographic Factors and Health Status}

Given the mortality rate, the chances of survival of the individuals with different demography is explored. For immediate policy interventions, identification of vulnerable demographic categories is critical, and this article estimated the likelihood of the presence or absence of bias in

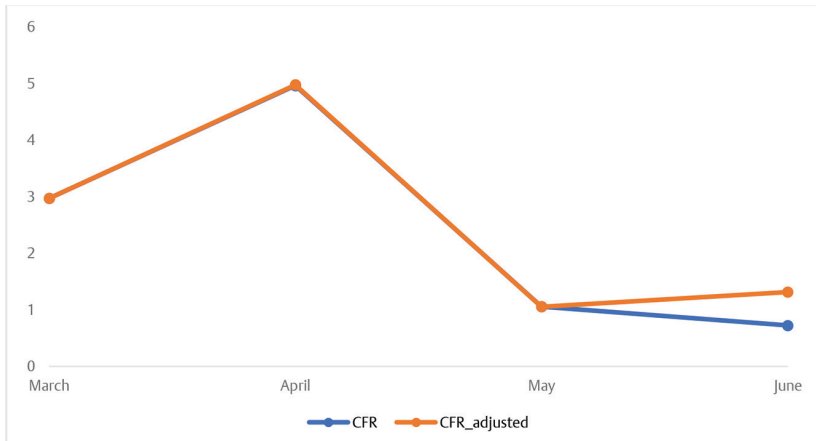

Fig. 5 Trends in month-wise case fatality rate (CFR) and adjusted case fatality rate (CFR*) for coronavirus disease 2019 (COVID-19) cases in Karnataka.

Table 4 Age- and gender-specific coronavirus disease 2019 (COVID-19) case fatality rate (CFR) and adjusted case fatality rate (CFR*) (in \%) in Karnataka, as of June 12, 2020

\begin{tabular}{|l|l|l|l|l|l|l|}
\hline Age groups $(\mathrm{y})$ & CFR-male & CFR $^{*}$-male & CFR-female & CFR $^{*}$-female & CFR-total & CFR $^{*}$-total \\
\hline $0-14$ & 0.00 & 0.00 & 0.22 & 0.32 & 0.10 & 0.14 \\
\hline $15-44$ & 0.22 & 0.32 & 0.19 & 0.28 & 0.21 & 0.31 \\
\hline $45-64$ & 2.67 & 3.81 & 4.44 & 6.33 & 3.24 & 4.63 \\
\hline $65+$ & 17.27 & 21.51 & 12.87 & 16.18 & 15.16 & 18.98 \\
\hline Total & 1.10 & 1.56 & 1.37 & 1.93 & 1.20 & 1.70 \\
\hline
\end{tabular}

Table 5 Geographical differences in coronavirus disease 2019 (COVID-19) fatality in Karnataka

\begin{tabular}{|l|l|l|l|l|l|l|}
\hline Geographical divisions & Total cases & Active & Deceased & Recovered & CFR & CFR $^{*}$ \\
\hline Geo 1 (Belgaum) & 891 & 273 & 12 & 606 & 1.35 & 1.76 \\
\hline Geo 2 (Gulbarga) & 2,422 & 1,308 & 19 & 1,095 & 0.78 & 1.21 \\
\hline Geo 3 (Mysuru) & 1,931 & 720 & 8 & 1,203 & 0.41 & 0.57 \\
\hline Geo 4 (Bengaluru) & 1,263 & 404 & 38 & 821 & 3.01 & 3.97 \\
\hline Geo 5 (from other states) & 23 & 1 & 1 & 21 & 4.35 & 4.54 \\
\hline
\end{tabular}

Abbreviations: CFR, case fatality rate; CFR*, adjusted case fatality rate. 
the recovery against fatality on the basis of gender, age, geographical location, or transmission type. Equation 3 has been estimated to explore the vulnerable categories.

The coefficients, as presented in - Table $\mathbf{6}$, measure the size of the log odds of an individual getting recovered against deceased from COVID-19 while moving from one category to the other. This is measured by deriving the exponential of the coefficient values from the original regression coefficients given in - Table 6, and the results are presented in - Table $\mathbf{7}$.

The coefficient of the intercept is much lower than 1 , and it depicts that the odds of recovery against deceased of an individual who is (belongs to base category) female, in the age group of 65 to 100 years, has a local transmission, and is an individual of other state but getting treated for COVID-19 in Karnataka is lower. In other words, it is found that the base category is more susceptible to the fatality as the odds of recovery are less. Further results are interpreted with reference to the base category, which remains constant throughout. With a change in the age of an individual from the base category to age 1 (0-14 years), the odds ratio of recovery against the deceased appears to fall to the lowest. If the chances of recovery of the population in the age group of 15 to 44 years are higher than the previous age group, it is even higher among the age group of 45 to 64 years.

A change in the type of transmission in the base category from local to import is the reflection of a drastic change in the odds of recovery. The findings indicate that keeping other levels in the base category constant, the odds of recovery against deceased of the person who has foreign travel history is higher than the individual with the history of local transmission.

The study also explores whether a differential location is a contributing factor in the recovery of COVID-19 patients. Equation 5 indicates that the odds ratio of recovery against deceased of an infected individual appears to be higher in geo
4 followed by geo 2 . Though it is low in geo 1 , it still appears to be closer to a satisfactory level. It could be inferred from the findings that the patients in geo 3 are exposed to higher risk and not only do the odds of recovery seem to be the lowest among all the regions but they are also much below the satisfactory.

Based on the exponential coefficient values discussed earlier, all possible combinations are explored and the odds ratio of their recovery against deceased is calculated and the findings corroborate with the outcome presented in -Table 7 and provide deeper insights. Even at the aggregate level, the odds of recovery against deceased among males are higher than females. Further, females who belong to age 1, local, and geo 1 are relatively more susceptible to fatality. The outcome reinforces the argument that males are more immune to health shocks than females. The comprehensive analysis depicts that among people in the age 4 and age 1 categories, the odds of being deceased appear to be more against recovery. In both the age categories, females are more susceptible than males. It has been found that the odds of recovery against being deceased increases among people in age 2 and it is even more in age 3.

Interestingly, the odds ratio of recovery against the deceased is higher among people who returned from foreign travel than the locally transmitted individuals. In contrast, this result is more pronounced among females than males. For instance, females of age 3 with import transmission, irrespective of geography they belonged to, have exhibited better odds of recovery against deceased than females with local transmission type. In other words, among males whether the transmission is by import or local, the odds of recovery against the deceased is the same. Quick response to the medical procedures, early detection, and better awareness among females would have contributed to this trend.

Table 6 Results of dummy variable logistic regression

\begin{tabular}{|l|l|l|l|l|}
\hline Regressor & Estimate coefficient & Std. error & z-Value & $\operatorname{Pr}(>|z|)$ \\
\hline (Intercept) & -0.6296 & 1.15975 & -0.543 & 0.587 \\
\hline Gender 1 & 0.06839 & 0.30034 & 0.228 & 0.82 \\
\hline Age 1 & -19.15194 & 767.8994 & -0.025 & 0.98 \\
\hline Age 2 & -4.8598 & 0.55552 & -8.748 & $<2 \mathrm{E}-16$ \\
\hline Age 3 & -1.55339 & 0.31326 & -4.959 & $7.09 \mathrm{E}-07$ \\
\hline Tans 1 & -1.03684 & 0.79929 & -1.297 & 0.195 \\
\hline Geo 1 & -0.9733 & 1.16898 & -0.833 & 0.405 \\
\hline Geo 2 & 1.14725 & -0.545 & 0.586 \\
\hline Geo 3 & -0.62545 & 1.18077 & -1.452 & 0.147 \\
\hline Geo 4 & -1.71405 & -0.327 & 0.744 \\
\hline
\end{tabular}

Table 7 Exponential of coefficient values measuring the chances of survival of the individuals with different demography

\begin{tabular}{|l|l|l|l|l|}
\hline Intercept & Gender 1 & Age 1 & Age 2 & Age 3 \\
\hline 0.5328075 & 1.070785 & $4.813021 \mathrm{e}-09$ & $7.752061 \mathrm{e}-03$ & 0.2115295 \\
\hline \multicolumn{5}{|l|}{} \\
\hline Trans 1 & Geo 1 & Geo 2 & Geo 3 & Geo 4 \\
\hline 0.3545744 & 0.3778334 & 0.5350218 & 0.1801345 & 0.6920511 \\
\hline
\end{tabular}


An aggregate analysis also exhibits that COVID-19 cases in geo 4 (Bengaluru division) and geo 2 (Gulbarga division) have an appreciable odds ratio of recovery, while it is moderate and satisfactory in geo 1 (Belgaum division). However, geo 3 (Mysuru division) and geo 5 (patients from out of state) are more susceptible to the risk of lower odds of recovery, confirming the result found from Equation 5. This implies that the recovery ratio differs from region to region and that could be attributed to various factors.

\section{Discussion}

This study was undertaken to understand the early transmission dynamics of COVID-19 in Karnataka, one of India's highest economic growth states with an expected gross state domestic product of US $\$ 258.37$ billion in the fiscal year $2020-21 .^{20}$ The trend analysis denotes that the number of COVID-19 cases in Karnataka was greatly suppressed during the lockdown period, but the state had seen an increase in the number of COVID-19 cases immediately after the relaxations in the lockdown norms. Karnataka was the first state in India to swiftly act with containment policy measures such as shutting down of schools, malls, theaters, and public-gathering events. Since March 24, 2020, Karnataka, with rest of India, was under lockdown at four different phases and each lockdown phase has varied with intensity and restrictions. ${ }^{21-23} \mathrm{Although}$ incidence in the occurrence of COVID-19 was apparent over time, growth was mild until the end of lockdown phase 3 (May 17). The early call of lockdown to maintain physical distancing as well as various prevention and control measures adopted by the state and central governments at all levels led to a reduction in the spread of COVID-19 disease outside the clusters. ${ }^{21,24,25}$ The lockdown and shut down of international airports have contributed to restraining further transmission of the infection from the people travel from abroad. However, since mid-May 2020 the government of India arranged special flights to airlift the citizens who were stranded abroad in a phased manner which has caused a rise in the infection getting transmitted to people travelled from abroad. The reported positive cases began to increase exponentially during phase 4 of the lockdown (May 18-May 31) and with unlock 1 from June 1, 2020. It intensifies the argument of whether people with foreign travel history were partly contributing to the contagious spread of the infection locally in Karnataka. During the early phase, the transmission of COVID-19 was urban-centric and limited to few districts such as Bengaluru Urban, Mysuru, Dakshina Kannada, and Kalburgi. However, during phase 4 of lockdown and unlock 1, mass migration of the stranded people from these regions accelerated the transmission of the virus even to the green regions and also to tier-2 and tier-3 cities, towns, and villages, causing a wide geospatial dispersion of the infection. The result was a rapid increase in positive cases.

A gender-based disparity in the susceptibility of COVID-19 cases with a male preponderance across all age groups has been explored. The observation made in this analysis corroborates with epidemiological data reported across different parts of the world where the majority (51-66.7\%) of affected patients had been male., ${ }^{3,26-28}$ There is a well-established gender-based difference in immune responses and differential susceptibility of men and women to infectious diseases. ${ }^{29,30}$ Several hypotheses have been proposed for the underlying reason behind the gender preponderance of COVID-19 infection, including increased expression of ACE-2, a male SARS-COV-2 binding receptor; sex-based immunological differences driven by sex hormone and the X-chromosome; and, eventually, gender inequality in the social activity and lifestyle-related behavior. ${ }^{28,31-33}$ However, further studies are warranted to elucidate the precise mechanism to understand the gender differences in the susceptibility of COVID-19 infection.

The early epidemiological studies on COVID-19 cases have shown that the susceptibility and risk of clinical symptoms following SARS-CoV-2 infection are age-dependent like other respiratory viral infections. ${ }^{34,35}$ The findings from the present study also corroborate the same. It is found that the adult population is more susceptible to the infection while it is low among children and elderly. Hence, the susceptibility to the infection has an inverted " $U$ " pattern of a relationship with respect to the age. Further, the study illustrates the geospatial dispersion of the COVID-19 cases and infers that substantial parts $(97 \%)$ of infected individuals are without travel history. Future studies need to validate whether people with foreign travel history were partly contributing to the contagious spread of the epidemic.

The early literature presumed that the risk of mortality from COVID-19 has a "Nike-Swoosh" pattern with high risk of mortality for children, lower risk among youths, and then increasing the risk for older adults and elderly and similar results were obtained by Joe and colleagues ${ }^{13}$ for Indian data covering till May 20, 2020. However, in contrast, the result of this study manifest that the burden of risk of mortality among children is near zero and it increases as we proceed to the higher age groups. Hence, the study derives an upwardly sloping curve from left to right. Further, our study explores that the youth age group has a higher burden of the infections, while the burden of death is relatively very high on the aged population. The finding of this study is consistent with the pattern of an age-based exponential increase in fatality rate as observed in other countries affected by COVID-19. ${ }^{36-38}$ Current global estimates of the clinical severity of COVID-19 indicate a determinant effect of age on the mortality of COVID-19 patients with a relevant age threshold of more than 50 years. ${ }^{39}$ Approximately $80 \%$ and $90 \%$ of deaths have occurred in patients aged $>70$ years and $\geq 60$ years in Korea and Italy, respectively. Although everyone has equal risk of getting infected to COVID-19 regardless of age or sex, the burden of fatality is unequally distributed, and it is more among the elderly population. It may be due to pre-existing medical conditions and the physiological aging process that lead to deterioration of health. A gender-wise analysis showed that the risk of fatality is higher among males than females. It is noteworthy that the risk of susceptibility to COVID-19 infection is lower among females. The study also identifies a wide regional disparity in the risk of 
fatality from COVID-19. The regional disparity in the CFR could be attributed to differences between the regions in various factors such as geoenvironment, urbanization, the intensity of connectivity and exposure to major cities, health infrastructure, awareness level, and economic development. It is also observed that CFR and adjusted CFR were diverging with time, indicating that Karnataka was in the early stage of transmission phase. The health administrators, hence, ought to consider the dynamics of CFR and adjusted CFR in designing and implementing epidemic containment strategies.

Though several studies have focused on the epidemiological analysis, and fatality analysis of COVID-19 in the context of different countries, none of them attended to identify the role of the demography of the infected person in determining his/her HS, that is, recovery or fatality. Since the outcome of such study is expected to contribute to the effective management of the pandemic, our study by estimating the logistic regression found that the demographic factors such as gender, age, transmission type, and geographical location of the infected individual contribute to his/her recovery from COVID-19. The chances of recovery of a female are lower than male, reinforcing that males are more immune to health shocks than females. Further, the chances of recovery are positively related to the age of the infected person. This demonstrates that chances of recovery are higher if the infected individual is younger, and the chances of recovery diminish if the individual is older. The findings reaffirm that the immune system of the elderly and the children is more vulnerable to the shocks of the virus. It is also ratified that the odds ratio of recovery against deceased increases with age as the human immune system grows stronger. The chances of recovery against deceased of the infected individual are also affected by his/her geographical location. It might be due to differential geoenvironment and socioeconomic environment. Other probable factors include regional differences in public awareness, random testing, early detection, early hospitalization after detection, and rehabilitation and early medical treatment post detection. It could also be due to wide regional differences in the availability of medical infrastructures such as hospitals, beds, medical staffs, testing devices, testing centers, ambulance services and transportation connectivity, and medical equipment like ventilators. Interestingly, the study also observes that the individuals who returned from foreign travel have better chances of recovery than the locally transmitted individuals. Though the risk of susceptibility to COVID-19 infection is equal to all, the burden of getting infected and the burden of fatality is unequally distributed among different demographic categories.

\section{Conclusion}

Deriving from the findings, the study arrives at the following inferences. First, individuals who are male, adult, and without foreign travel are more susceptible to COVID-19. Second, female and elderly individuals have a higher risk of fatality. Third, there exist regional differences as well in susceptibility to COVID-19 and fatality from this epidemic. Four, infected individuals who are male, young, and returned from foreign countries have higher chances of recovery from the infection. Thus, to conclude, though the burden of risk of infection to COVID-19 is the same to all, the burden of fatality and chances of recovery are unequally distributed among different demographic categories. To manage the contagious transmission of the epidemic, to reduce fatality, and to increase the chances of recovery, targeted policy actions are inevitable. Hence, the findings of the study have significant policy implications. Since the pandemic is underway, the findings of the study cannot be generalized and rather should be confined to the study region and study period. To the best of our knowledge, this is the first effort in unraveling the contribution of different categories of demographic factors to the HS of the infected individuals. Further studies may focus on whether the dynamics do change with the increased cases and in different geography.

\section{Conflict of Interest}

None declared.

\section{Acknowledgments}

The authors are grateful to all the data contributors of the COVID-19 India crowd-sourced initiative.

\section{References}

1 Dawood FS, Ricks P, Njie GJ, et al. Observations of the global epidemiology of COVID-19 from the prepandemic period using web-based surveillance: a cross-sectional analysis. Lancet Infect Dis 2020;20(11):1255-1262

2 Situation Report WHO. 2020. https://apps.who.int/iris/handle/10665/333905. Accessed March 9, 2021

3 Huang C, Wang Y, Li X, et al. Clinical features of patients infected with 2019 novel coronavirus in Wuhan, China. Lancet 2020;395(10223) :497-506

4 Hu B, Guo H, Zhou P, Shi ZL. Characteristics of SARS-CoV-2 and COVID-19. Nat Rev Microbiol 2021;19(3):141-154

5 Guan WJ, Ni ZY, Hu Y, et al; China Medical Treatment Expert Group for Covid-19. Clinical characteristics of coronavirus disease 2019 in China. N Engl J Med 2020;382(18):1708-1720

$6 \mathrm{Gu}$ J, Korteweg C. Pathology and pathogenesis of severe acute respiratory syndrome. Am J Pathol 2007;170(4):1136-1147

7 Dhama K, Patel SK, Pathak M, et al. An update on SARSCoV-2/COVID-19 with particular reference to its clinical pathology, pathogenesis, immunopathology and mitigation strategies. Travel Med Infect Dis 2020;37:101755

8 Andrews MA, Areekal B, Rajesh KR, et al. First confirmed case of COVID-19 infection in India: A case report. Indian J Med Res 2020;151(5):490-492

9 Yadav PD, Potdar VA, Choudhary ML, et al. Full-genome sequences of the first two SARS-CoV-2 viruses from India. Indian J Med Res 2020;151(2 \& 3):200-209

10 Khan MI, Khan ZA, Baig MH, et al. Comparative genome analysis of novel coronavirus (SARS-CoV-2) from different geographical locations and the effect of mutations on major target proteins: An in silico insight. PLoS One 2020;15(9):e0238344

11 prpt. Kumar BK, Venkatraja B, Prithvisagar KS, et al. Mutational analysis unveils the temporal and spatial distribution of G614 genotype of SARS-CoV-2 in different Indian states and its association with case fatality rate of COVID-19.bioRxiv 2020. DOI: 10.1101/2020.07.27.222562/prpt

12 Parlikar A, Kalia K, Sinha S, et al. Understanding genomic diversity, pan-genome, and evolution of SARS-CoV-2. PeerJ 2020;8:e9576 
13 Joe W, Kumar A, Rajpal S, Mishra US, Subramanian SV. Equal risk, unequal burden? Gender differentials in COVID-19 mortality in India. J Glob Health Sci 2020;2(1). Doi: 10.35500/jghs.2020.2.e17

14 Lipsitch M, Donnelly CA, Fraser C, et al. Potential biases in estimating absolute and relative case-fatality risks during outbreaks. PLoS Negl Trop Dis 2015;9(7):e0003846

15 Russell TW, Hellewell J, Jarvis Cl, et al; Cmmid Covid-Working Group. Estimating the infection and case fatality ratio for coronavirus disease (COVID-19) using age-adjusted data from the outbreak on the Diamond Princess cruise ship, February 2020. Euro Surveill 2020;25(12):2000256

16 Garg S, Kim L, Whitaker M, et al. Hospitalization rates and characteristics of patients hospitalized with laboratory-confirmed coronavirus disease 2019-COVIDNET, 14 States, March 1-30, 2020. MMWR 2020; 69(15):458-464

17 Guo YR, Cao QD, Hong ZS, et al. The origin, transmission and clinical therapies on coronavirus disease 2019 (COVID-19) outbreak-an update on the status. Mil Med Res 2020;7(1):1-0

18 Vigo D, Thornicroft G, Gureje 0 . The differential outcomes of coronavirus disease 2019 in low- and middle-income countries vs high-income countries. JAMA Psychiatry 2020; 77(12):1207-1208

19 Mi YN, Huang TT, Zhang JX, et al. Estimating the instant case fatality rate of COVID-19 in China. Int J Infect Dis 2020;97:1-6

20 IBEF-India Brand Equity Foundation newsletter on industrial development and economic growth in Karnataka. 2020. https://www.ibef.org/states/karnataka-presentation. Accessed March 9, 2021

21 The Lancet. India under COVID-19 lockdown. Lancet 2020; 395(10233): 1315

22 Chetterje P. Gaps in India's preparedness for COVID-19 control. Lancet Infect Dis 2020;20(5):544

23 Sardar T, Nadim SS, Rana S, Chattopadhyay J. Assessment of lockdown effect in some states and overall India: A predictive mathematical study on COVID-19 outbreak. Chaos Solitons Fractals 2020;139:110078

24 Mahajan P, Kaushal J. Epidemic trend of COVID-19 transmission in India during lockdown-1 Phase. J Community Health 2020; 45(6):1291-1300

25 Mandal S, Bhatnagar T, Arinaminpathy N, et al. Prudent public health intervention strategies to control the coronavirus disease 2019 transmission in India: A mathematical model-based approach. Indian J Med Res 2020;151(2 \& 3):190-199
26 Walter LA, McGregor AJ. McGregorAJ. Sex- and gender-specific observations and implications for COVID-19. West J Emerg Med 2020;21(3):507-509

27 Smith J, Griffith D, White A, et al. COVID-19, equity and men's health. Int J Mens Soc Comm Health. 2020;3(1):e48-e64

28 Jin JM, Bai P, He W, et al. Gender differences in patients with COVID-19: focus on severity and mortality. Front Public Health 2020;8:152

29 Klein SL, Flanagan KL. Sex differences in immune responses. Nat Rev Immunol 2016;16(10):626-638

30 Scully EP, Haverfield J, Ursin RL, Tannenbaum C, Klein SL. Considering how biological sex impacts immune responses and COVID-19 outcomes. Nat Rev Immunol 2020;20(7):442-447

31 Bwire GM. Coronavirus: why men are more vulnerable to COVID-19 than women? SN Compr Clin Med 2020;7:1-3

32 Foresta C. Rocca MS, Di Nisio A. Gender susceptibility to COVID-19: a review of the putative role of sex hormones and X chromosome. J Endocrinol Invest 2020;16:1-6

33 Kopel J, Perisetti A, Roghani A, Aziz M, Gajendran M, Goyal H. Racial and gender-based differences in COVID-19. Front Public Health 2020;8:418

34 Davies NG, Klepac P, Liu Y, Prem K, Jit M, Eggo RM; CMMID COVID-19 working group. Age-dependent effects in the transmission and control of COVID-19 epidemics. Nat Med 2020;26(8):1205-1211

35 Dong Y, Mo X, Hu Y, et al. Epidemiology of COVID-19 among children in China. Pediatrics 2020;145(6):e20200702

36 Heald-Sargent T, Muller WJ, Zheng X, et al. Age-related differences in nasopharyngeal severe acute respiratory syndrome coronavirus 2 (SARS-CoV-2) levels in patients with mild to moderate coronavirus disease 2019 (COVID-19). JAMA Pediatr 2020;174(9):902-903

37 Onder G, Rezza G, Brusaferro S. Case-fatality rate and characteristics of patients dying in relation to COVID-19 in Italy. JAMA 2020;323(18):1775-1776

$38 \mathrm{Wu} \mathrm{Z}$, McGoogan JM. Characteristics of and important lessons from the coronavirus disease 2019 (COVID-19) outbreak in China: summary of a report of 72314 cases from the Chinese Center for Disease Control and Prevention. JAMA 2020;323(13):1239-1242

39 Bonanad C, García-Blas S, Tarazona-Santabalbina F, et al. The effect of age on mortality in patients with Covid-19: a metaanalysis with 611,583 subjects. J Am Med Dir Assoc 2020;21(7):915-918 